\title{
Craniofacial Statistical Deformation Models of Wild-type mice and Crouzon mice
}

\author{
Hildur Ólafsdóttir ${ }^{*} a, b$, Tron A. Darvann ${ }^{b}$ Bjarne K. Ersbøll $^{a}$, Nuno V. Hermann ${ }^{b, c}$, Estanislao Oubel ${ }^{d}$, Rasmus \\ Larsen $^{a}$, Alejandro F. Frangi $d$, Per Larsen ${ }^{b}$, Chad A. Perlyn ${ }^{e}$, Gillian M. Morriss-Kay $f$, Sven Kreiborg $b, c, g$ \\ $a_{\text {Informatics and Mathematical Modelling, Technical University of Denmark, Denmark }}$

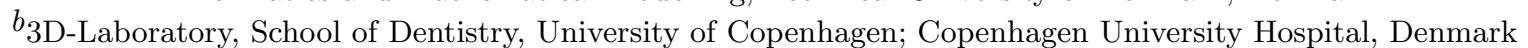 \\ ${ }^{c}$ Department of Pediatric Dentistry and Clinical Genetics, School of Dentistry, Faculty of Health Sciences, University of \\ Copenhagen, Denmark

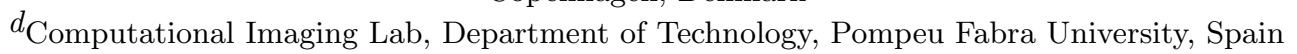 \\ ${ }^{e}$ Division of Plastic Surgery, Washington University School of Medicine, USA

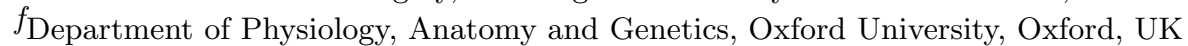

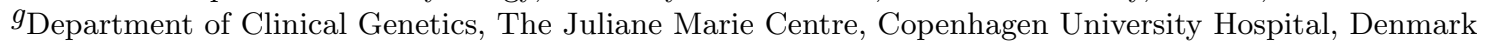

\begin{abstract}
Crouzon syndrome is characterised by premature fusion of cranial sutures and synchondroses leading to craniofacial growth disturbances. The gene causing the syndrome was discovered approximately a decade ago and recently the first mouse model of the syndrome was generated. In this study, a set of Micro CT scans of the heads of wild-type (normal) mice and Crouzon mice were investigated. Statistical deformation models were built to assess the anatomical differences between the groups, as well as the within-group anatomical variation. Following the approach by Rueckert et al. we built an atlas using B-spline-based nonrigid registration and subsequently, the atlas was nonrigidly registered to the cases being modelled. The parameters of these registrations were then used as input to a PCA. Using different sets of registration parameters, different models were constructed to describe (i) the difference between the two groups in anatomical variation and (ii) the within-group variation. These models confirmed many known traits in the wild-type and Crouzon mouse craniofacial anatomy. However, they also showed some new traits.
\end{abstract}

Keywords: Statistical Deformation Model, Nonrigid Registration, Atlas, Crouzon Syndrome, Mouse Model, Micro CT, Craniofacial

\section{INTRODUCTION}

Crouzon syndrome was first described nearly a century ago when calvarial deformities, facial anomalies, and abnormal protrusion of the eyeballs were reported in a mother and her son. ${ }^{1}$ Later it was found that the condition was characterised by varying degree of synostosis of sutures of the cranium, the cranial base, the orbits and the maxilla, sometimes combined with premature closure of the synchondroses of the cranial base. The phenotype is characterised by abnormal cranial shape, shallow orbits, maxillary hypoplasia, beaked nose, crowding of teeth and higharched or cleft-palate. Heterozygous mutations in the gene encoding fibroblast growth factor receptor type 2 (FGFR2) have been found responsible for Crouzon syndrome. ${ }^{2}$ Recently a mouse model was created to study one of these mutations $\left(F G F R 2^{C y s 342 T y r}\right){ }^{3}$ Incorporating advanced small animal imaging techniques such as Micro CT, allows for detailed examination of the craniofacial growth disturbances. Studying the craniofacial shape differences in detail contributes to the understanding of the syndrome, surgery planning and diagnosis in humans. A recent study, performing linear measurements on Micro CT scans, proved the mouse model applicable to reflect the craniofacial deviations occurring in humans with Crouzon syndrome. ${ }^{4}$ Previously, we have extended this study to assess the local deformations between the groups by constructing a deformable shape and intensity-based atlas of wild-type (normal) mouse skulls. Deforming this atlas to all mice, the craniofacial shape differences were analysed. ${ }^{5}$

In order to analyse and interpret these deformations in a meaningful way, it is desirable to reduce the large number of dimensions and at the same time localise the deviations with respect to the atlas. This leads us to

\footnotetext{
*Corresponding author: email: ho@imm.dtu.dk, telephone: +45 45253422.
} 
Statistical Deformation Models (SDMs). These are closely related to statistical shape models but the fact that the whole correspondence field is modelled makes them more powerful.

The focus of this paper will be to use the SDMs to (i) describe the anatomical variability between the two groups, i.e. extract the differences, and (ii) describe the anatomical variability within each of the two groups.

\section{DATA MATERIAL}

Production of the $F g f r 2^{C 342 Y /+}$ and $F g f r 2^{C 342 Y / C 342 Y}$ mutant mouse (Crouzon mouse) has been previously described. ${ }^{3}$ All procedures were carried out in agreement with the United Kingdom Animals (Scientific Procedures) Act, guidelines of the Home Office, and regulations of the University of Oxford.

For three-dimensional (3D) CT scanning, 10 wild-type and $10 \mathrm{Fg} f r 2^{C 342 Y /+}$ specimens at six weeks of age (42 days) were sacrificed using Schedule I methods and fixed in $95 \%$ ethanol. They were sealed in conical tubes and shipped to the Micro CT imaging facility at the University of Utah. Images of the skull were obtained at approximately $46 \mu \mathrm{m} \times 46 \mu \mathrm{m} \times 46 \mu \mathrm{m}$ resolution using a General Electric Medical Systems EVS-RS9 Micro CT scanner. Prior to analysis of the images the mandible was masked out for all 20 specimens. This was due to different jaw positions and the fact that the deviation in mandible shape is a secondary effect of the syndrome. Figure 1 shows an example of the living mice and the imaging data appearance.

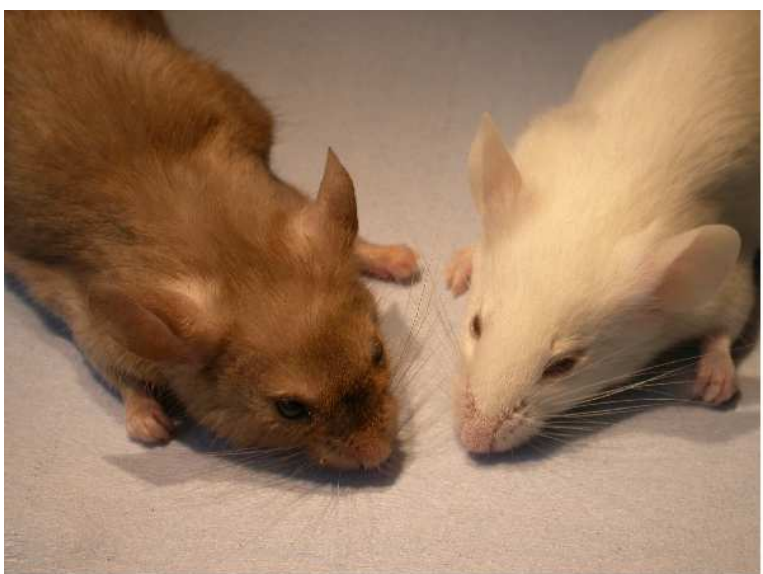

(a)

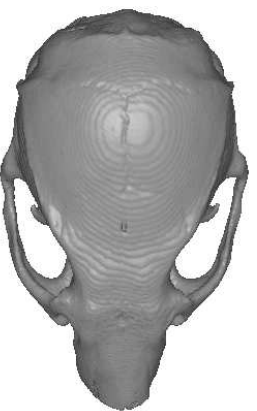

(b)

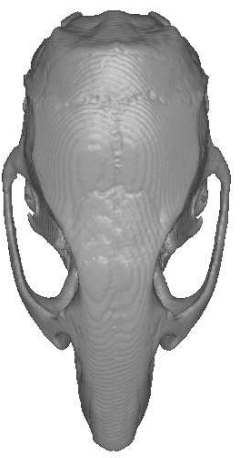

(c)

Figure 1. (a) Photo of a Crouzon mouse (left) and a wild-type mouse (right). Skulls extracted from CT images of (b) a Crouzon mouse, (c) a wild-type mouse.

\section{METHODS}

The steps taken to automatically build a SDM are the following.

1. Build an atlas from a set of images using nonrigid image registration.

2. Match the atlas to the cases to be included in the model using nonrigid image registration.

3. Build a Statistical Deformation Model applying a PCA to the resulting deformation fields.

\subsection{Registration and Atlas Building}

For steps 1 and 2 above, the nonrigid registration algorithm based on B-splines ${ }^{6,7}$ was applied. This algorithm uses a transformation model which is a combination of a global and a local transformation model,

$$
\mathbf{T}(\mathbf{x})=\mathbf{T}_{\text {global }}(\mathbf{x})+\mathbf{T}_{\text {local }}(\mathbf{x}) .
$$


The global transformation model consists of an affine transformation matrix. The local transformation model describing the nonrigid part of the model is written by the tensor product of $1 \mathrm{D}$ cubic B-splines,

$$
\mathbf{T}_{\text {local }}(x, y, z)=\sum_{l=0}^{3} \sum_{m=0}^{3} \sum_{n=0}^{3} B_{l}(u) B_{m}(v) B_{n}(w) \mathbf{c}_{i+l, j+m, k+n}
$$

where $\mathbf{c}$ are the parameters (control points) of the B-splines ordered in a $p_{x} \times p_{y} \times p_{y}$ lattice. $i, j$ and $k$ are the control point positions and $u, v$ and $w$ are the $(x, y, z)$ image coordinates transformed into the lattice coordinates.

\subsection{Statistical Deformation Model}

Statistical deformation models are closely related to statistical shape models. The key difference is that shape models require shapes represented by points which correspond over the set. This is dependent on manual labour although many authors have suggested approaches to reduce this. ${ }^{8-10}$ Statistical deformation models aim at modelling the deformation fields acquired by the set of nonrigid registrations, each initiated in the common reference (atlas image or a selected image from the set to be modelled). In this way, anatomical variability in the full volume is obtained, not only in a segmented shape.

In this paper, we follow the approach by Rueckert et al. ${ }^{11}$ They take advantage of the fact that the parameters of the B-splines (the control points) compactly represent the correspondence field between the atlas and the target. After removing influence from the global model by multiplying the control points by the inverse Jacobian of $T_{\text {global }}$, the control points can be used as input to PCA in order to model the deformations of the group of subjects defined in the domain of the atlas. Concatenating the 3D control points for subject $s$ into a row vector $\mathbf{C}_{s}=\left[c_{1}, \ldots, c_{p}\right]$, where $p=3 p_{x} p_{y} p_{z}$, gives the $p$ th row of the $n \times p$ data matrix to analyse $(n$ is the number of observations). We define the mean deformation

$$
\overline{\mathbf{C}}=\frac{1}{n} \sum_{s=1}^{n} \mathbf{C}_{s}
$$

and the covariance matrix

$$
\frac{1}{n-1} \sum_{s=1}^{n}\left(\mathbf{C}_{s}-\overline{\mathbf{C}}\right)\left(\mathbf{C}_{s}-\overline{\mathbf{C}}\right)^{T} .
$$

An eigenanalysis on the covariance matrix gives the matrix of eigenvectors $\boldsymbol{\Phi}$ and the corresponding eigenvalues, $\lambda$. Now, a new control point instance can be generated by the model,

$$
\hat{\mathbf{C}}=\overline{\mathbf{C}}+\Phi \mathbf{b} .
$$

Typically, the number of eigenvectors, $t$, included in the model is chosen so that that they explain at least $95 \%$ of the variance in the model. Now $\mathbf{b}$ is the t-dimensional vector of model parameters.

\subsection{Modelling Approaches}

In order to describe as compactly and descriptively as possible the different types of variations of interest, the types of atlases and transformation models in the registrations need to be considered.

To model the differences between groups, we use a wild-type mouse atlas, built from the group of wild-type mice. Depending on the kind of variation we are interested in modelling, we use different global transformation models $\left(T_{\text {global }}\right)$ in the registrations. In our previous study, we used nine degrees of freedom (rotation, translation and anisotropic scaling), noting that the largest differences between the groups occur in skull length, width and height. ${ }^{5}$ The local model in this case provides the local differences between the groups left, when height, width and length have been removed. This gives us an interesting view of the group differences, but is perhaps not very intuitive. Including height, width and length differences in the local model gives a more intuitive model. Hence, the registrations were repeated using a global model with six degrees of freedom (rotation and translation) and a local model covering the height, width and length differences. We will refer to the two different combined local models as model A and model B, with height, width and length differences excluded and included, respectively. 
In order to describe the within-group variation compactly, an atlas descriptive of the group in question was used as a reference (source) for the registrations (the wild-type atlas for the wild-type mouse model and a Crouzon atlas for the Crouzon mouse model). Seven degrees of freedom were used in the global model, i.e. translation, rotation and an isotropic scaling. Hence, the effect of pose and size was removed from the local model. We could possibly have used the deformations of model A, but this was not considered appropriate since there is no hypothesis stating that within-group variation systematically involves anisotropic scaling differences. Table 1 summarises the different modelling approaches.

Table 1. Modelling approaches

\begin{tabular}{|l|l|l|l|}
\hline Model & Source of registration & DOF in $T_{\text {global }}$ & Covered by local model \\
\hline $\mathrm{A}$ & Wild-type atlas & 9 & Local deformations \\
$\mathrm{B}$ & Wild-type atlas & 6 & Height, length and width, local deformations \\
Wild-type & Wild-type atlas & 7 & Local deformations \\
Crouzon & Crouzon atlas & 7 & Local deformations \\
\hline
\end{tabular}

\section{EXPERIMENTAL RESULTS}

Each type of registration from Table 1 was carried out hierarchically, using local models with control point spacing of 3, 1.5 and $0.75 \mathrm{~mm}$ for model $\mathrm{A}$ and the within-group models. For model B, control point spacing of $6,3,1.5$ and $0.75 \mathrm{~mm}$ for model $\mathrm{B}$ was applied since the length differences required more than $3 \mathrm{~mm}$ spacing to be covered by the local transformation only.

\subsection{Registration Accuracy}

Sufficient accuracy of the image registration algorithm is essential for the deformation model to be valid. In our previous work, manual annotations from two observers were used to assess the registration accuracy of model A. Using the optimal transformations from the image registrations, landmarks were obtained automatically. The landmark positions were statistically compared to those annotated by the human observers. This showed that the automatic method provided as good accuracy as the human observers and moreover, it was more precise, judged from the significantly lower standard deviation. ${ }^{5}$ Following the same approach for model B, Figure 2 shows the landmark errors for 26 landmarks set by the two observers as compared to the automatically obtained landmarks. We show this for Crouzon cases only since it is considered a more challenging task. For the wild-type cases even better results are obtained. The plots are scaled to obtain reasonable comparisons as suggested in our previous work. ${ }^{5}$ Statistical comparison of the landmark position revealed the same main conclusions as the ones from the previous study on model A. Additionally, similar results were obtained for the within-group models. In conclusion, we have established a good basis for reliable deformation models.

\subsection{SDM of Crouzon and Wild-type Mice}

A SDM was built for both types of local transformation, i.e. without and with (anisotropic) scaling differences (model A and model B). In each case, a PCA was applied to the matrix of control points with $n=20$ observations. For model A, $p=15525$ and for model B, $p=21675$. For model A, 15 principal modes were required to retain $95 \%$ of the variation in local deformation from average anatomy. For model B, on the other hand, 10 principal modes were required. A plot of the cumulative variance across modes for each model is shown in Figure 3. The observations, projected into the space of the first six modes of variation, are shown in Figure 4 for models A, and B.

Figures 5 and 6 show the first mode of variation for models A and B, respectively shown by extracting the skull surfaces from the deformed images created by the models. This mode is discriminating for the two groups. 


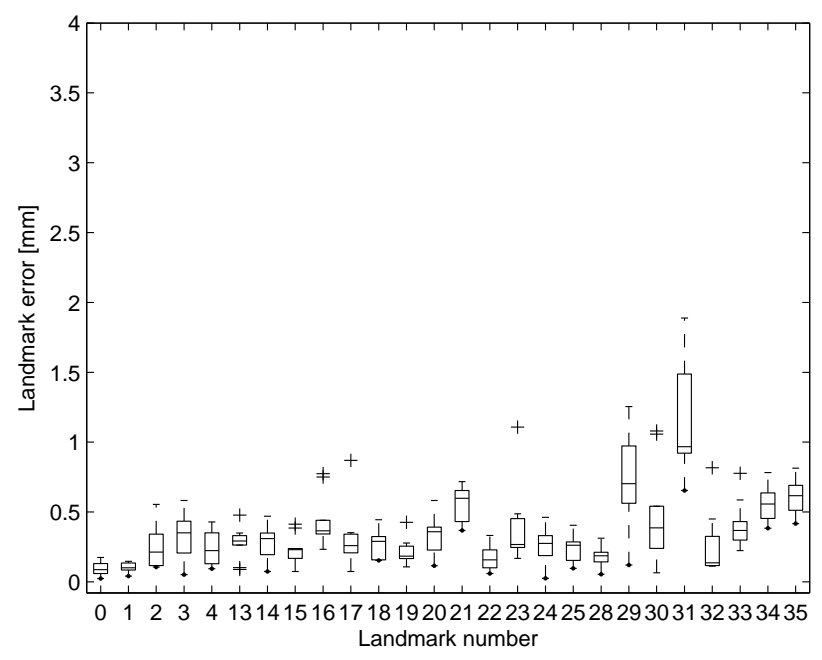

(a)

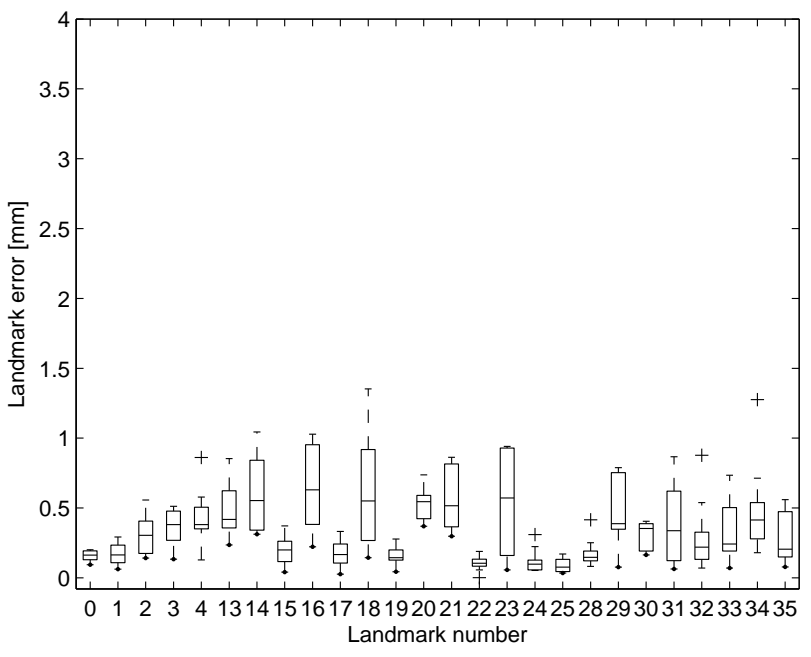

(b)

Figure 2. (a) Landmark errors for Crouzon mice between (a) automatic approach using model B and gold standard; (b) observer I and observer II.

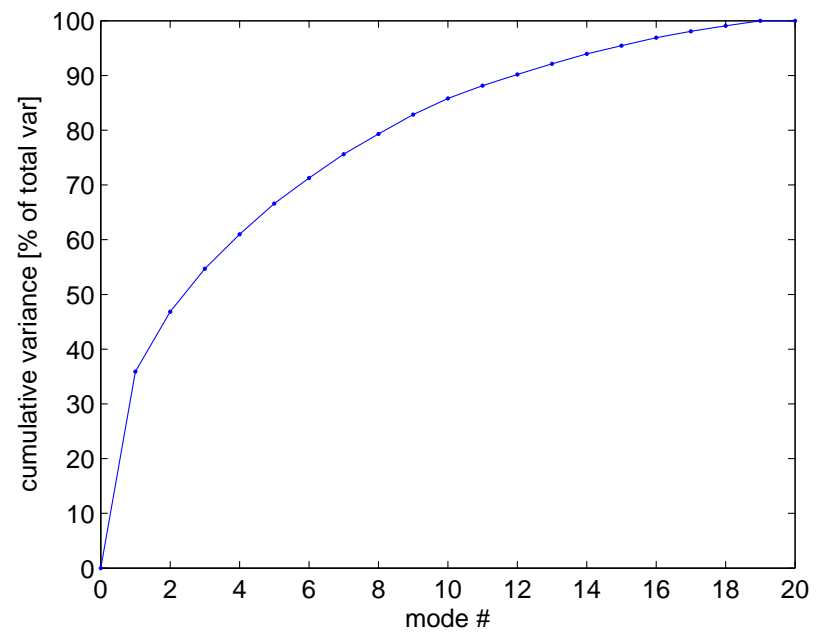

(a)

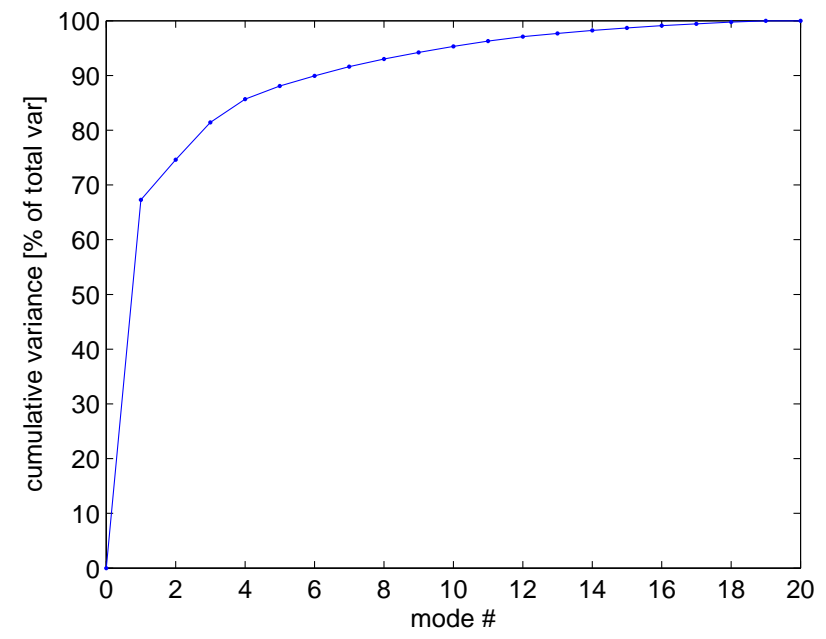

(b)

Figure 3. Cumulative variance across the modes of the statistical deformation model. (a) Model A (b) Model B .

\subsection{Within-group SDMs}

A SDM of the wild-type mouse anatomy was built using the control point vectors from the wild-type local model, which form a data matrix with $n=10$ and $p=15525$. Eight modes of variation were required to capture $95 \%$ of the total variance. Figure 7 shows the first and second mode of variation in the wild-type mouse craniofacial SDM.

A similar data matrix was obtained from the Crouzon local model. The craniofacial Crouzon SDM required eight modes of variation to capture $95 \%$ of the total variance.

\section{DISCUSSION}

Figure 3 shows that the main difference between model A and B is the proportion of variance explained by the first mode. As expected, the first mode of model B explains a large proportion of variance, since the length, width and height differences are considerable. Figure 4 shows that for both of the combined models (A and B), 


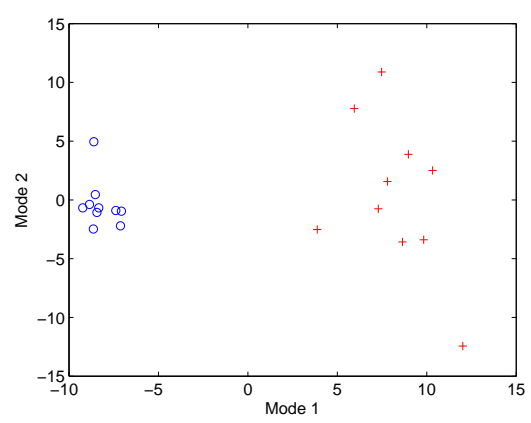

(a)

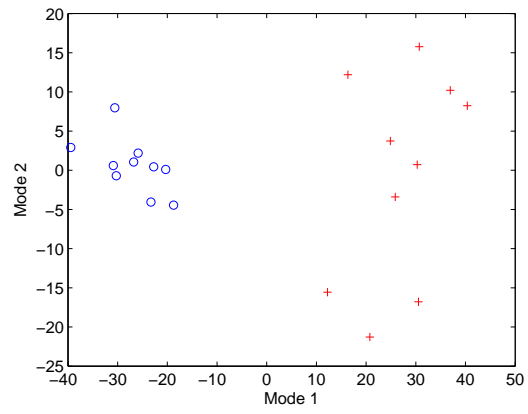

(d)

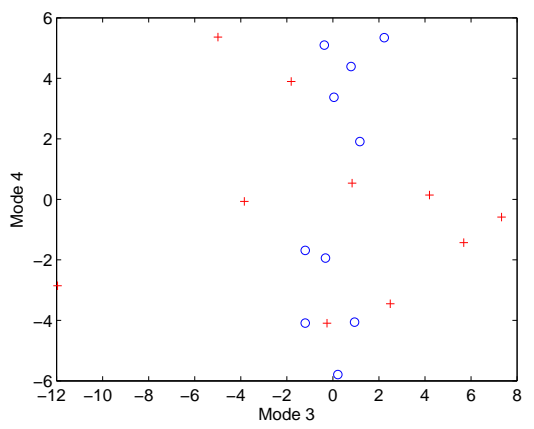

(b)

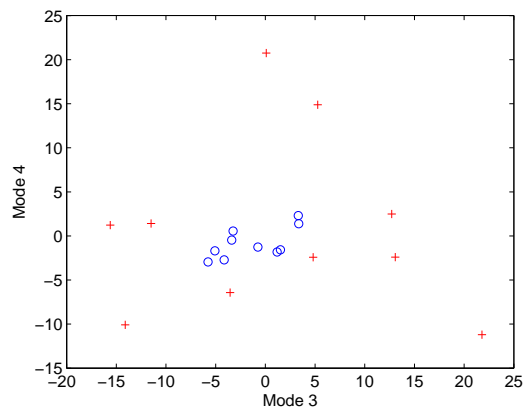

(e)

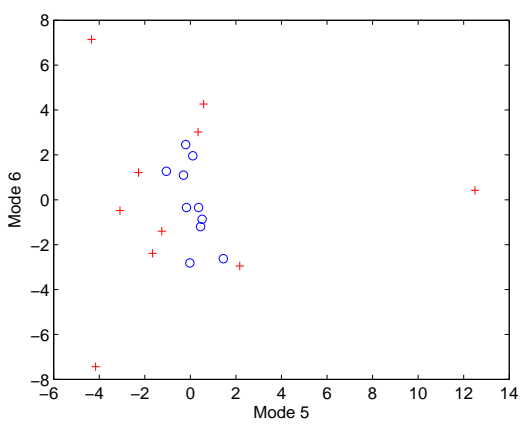

(c)

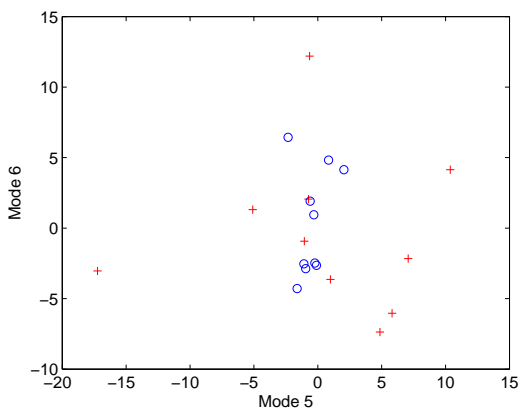

(f)

Figure 4. Projection of observations into the space of the first six principal components for model A (a-c) and model B (d-f). Crosses denote Crouzon cases while circles denote wild-type cases. (a,d) Mode 2 vs. mode 1 ; (b,e) Mode 4 vs. mode $3 ;(\mathrm{c}, \mathrm{f})$ Mode 6 vs. mode 5 .

only the first mode of variation is discriminating for the two groups. The remaining modes describe other types of variation than the one related to group differences. It is also noted from this figure that the variability in the Crouzon mouse group is for most modes considerably larger than for wild-type cases. This is often the case when comparing a control group to a diseased group.

For model A, it is noted that in the Crouzon case, the maxillary molar teeth are located posteriorly relative to the wild-type (see Figure 5, (c,d) causing a malocclusion. Additionally, the Crouzon mice have a shorter cranial base (see Figure 5, (c,d)), a shorter nose (as measured from sutura frontonasalis, see Figure 5, (a,b)). These three traits have earlier been observed in human subjects. ${ }^{12}$ Furthermore, this model describes a constriction of the cranial base, meaning that while the calvaria becomes higher, the cranial base becomes shorter and over bent (see Figure 5, (c-f)). This confirms a result from our previous study where the deformation field between a Crouzon mouse atlas to the wild-type mouse atlas was inspected. ${ }^{5}$ This interesting effect has not been reported before, and calls for a further investigation. As expected, the largest variation in model B is due to differences in length, height and width. Nevertheless, the previously mentioned tendencies in the cranial base can be observed.

The fact that only the first mode of variation differentiates between the groups makes the model more difficult to interpret and probably some differences between the groups are not represented in this mode. Ongoing and future work is therefore to use more powerful techniques than PCA to distribute the variation across modes.

The wild-type mouse SDM in Figure 7 indicates that wild-type mice vary in shape of the zygoma (Figure 7(a,b)) and the curvature of the calvaria and nose bending (Figure $7(\mathrm{c}, \mathrm{d})$ ).

The Crouzon SDM in Figure 8 shows that Crouzon mice vary in shape and asymmetry of zygoma (Figure 8 $(a, b))$. Judged from the third mode, Crouzon mice have different degree of curved calvaria and bending of the nose (Figure $8(\mathrm{c}, \mathrm{d})$ ). The sixth mode mainly describes the orientation and asymmetry of the nose (Figure 8 $(e, f))$. The asymmetric behavior noted in the second and sixth mode (and more not shown here) is understandable due to the syndrome's nature of full or partial cranial suture fusing, at different sides of the skull. The fact that 


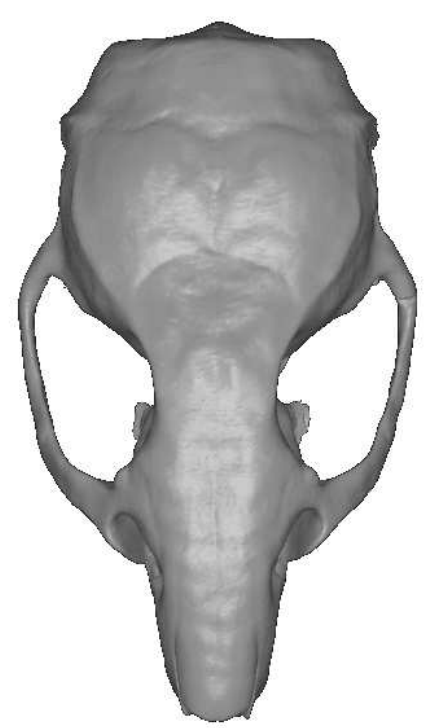

(a)

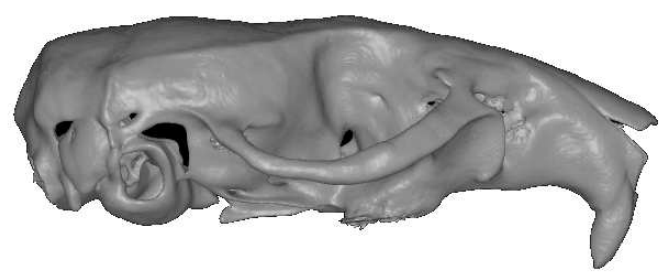

(c)

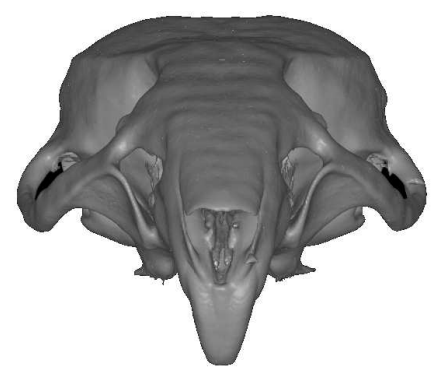

(e)

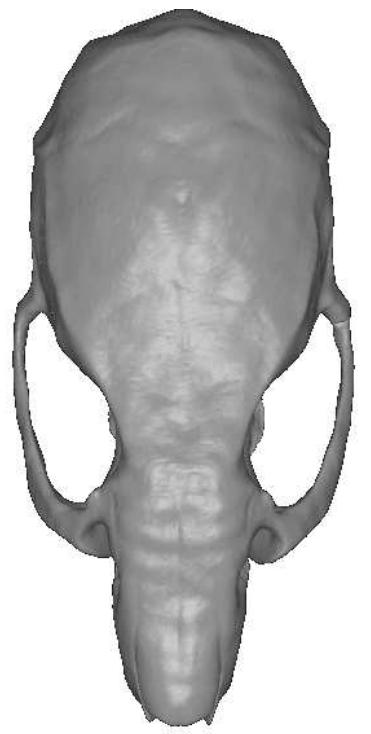

(b)

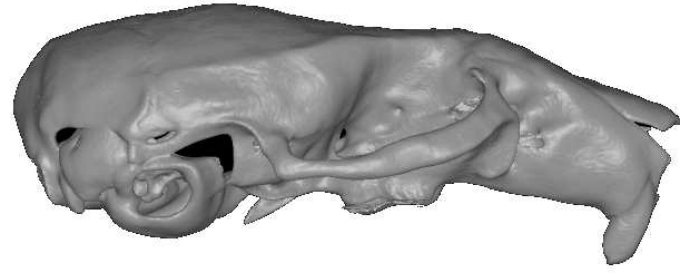

(d)

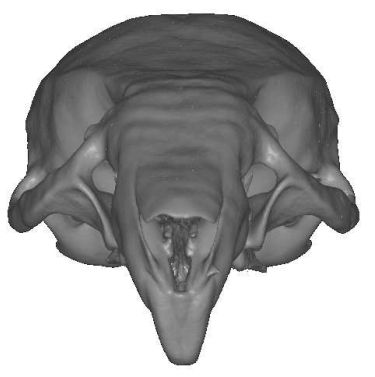

(f)

Figure 5. First mode of deformation variation, after removing height, length and width differences (model A). (a,c,e) Atlas deformed towards the most extreme wild-type case, (b,d,f) Atlas deformed towards the most extreme Crouzon case. $(\mathrm{a}, \mathrm{b})$ : top view; $(\mathrm{c}, \mathrm{d})$ : side view; $(\mathrm{e}, \mathrm{f})$ : front view.

both wild-type mice and Crouzon mice vary in shape of zygoma and curving of calvaria indicates that this is a normal, biological variation. However, the Crouzon variation is more extreme, which is in agreement with the previous observation of large variability in the Crouzon group. 


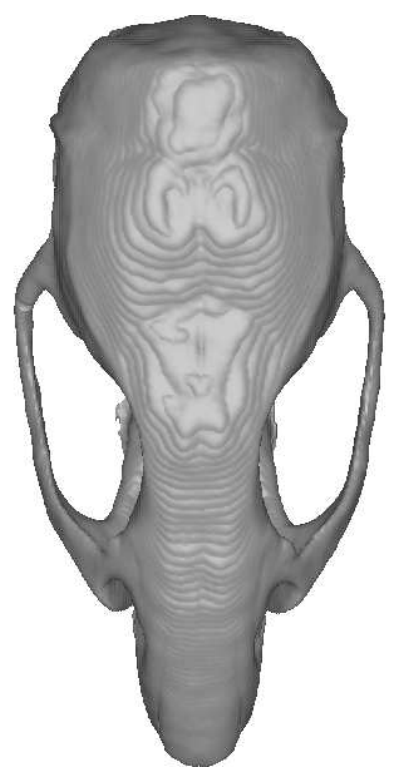

(a)

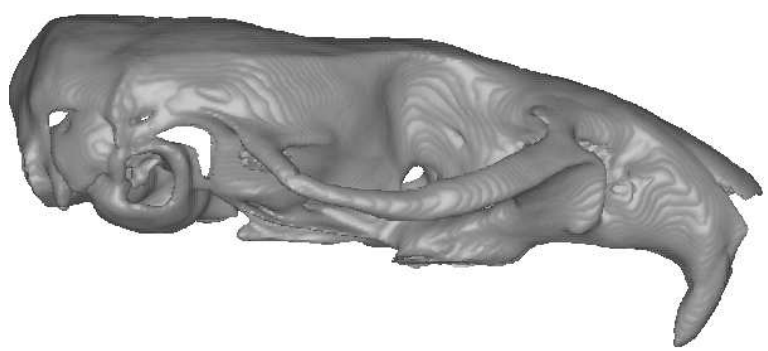

(c)

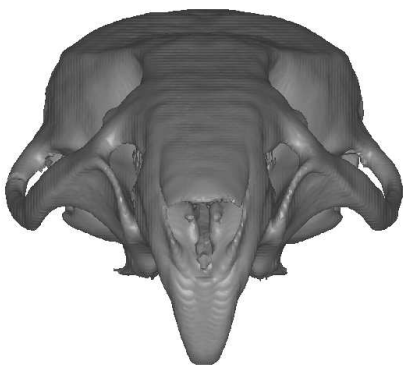

(e)

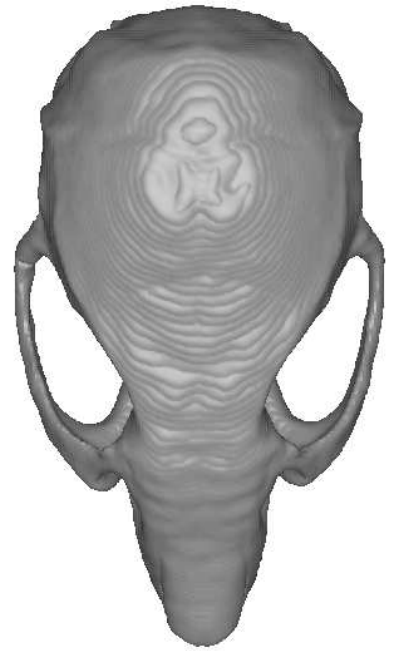

(b)

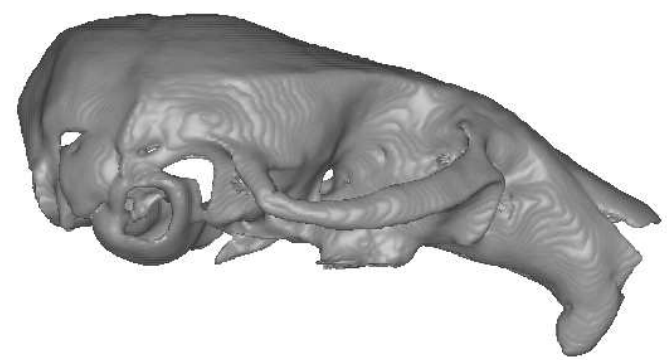

(d)

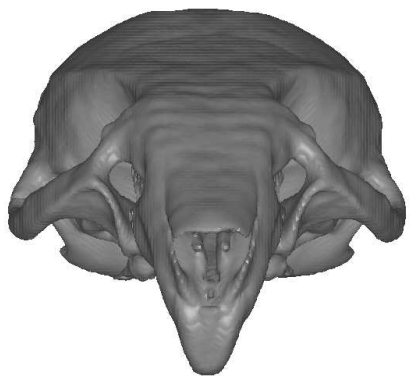

(f)

Figure 6. First mode of deformation variation, with height, width and length included in the local model (model B) $(\mathrm{a}, \mathrm{c}, \mathrm{e})$ Atlas deformed towards the most extreme wild-type case, (b,d,f) Atlas deformed towards the most extreme Crouzon case. $(\mathrm{a}, \mathrm{b})$ : top view; $(\mathrm{c}, \mathrm{d})$ : side view; $(\mathrm{e}, \mathrm{f})$ : front view. 


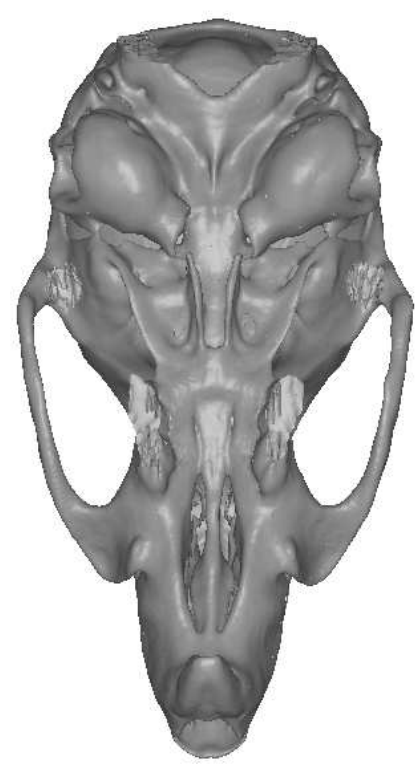

(a)

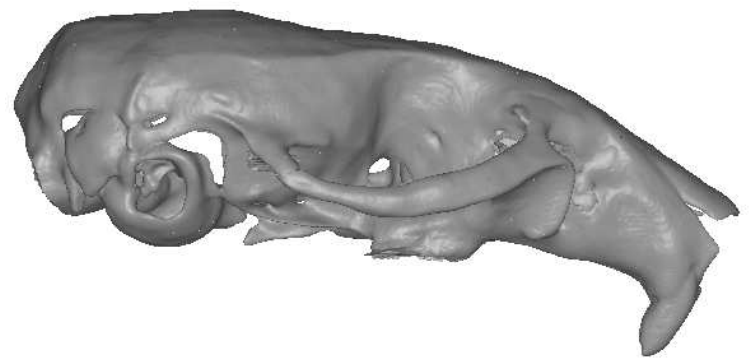

(c)

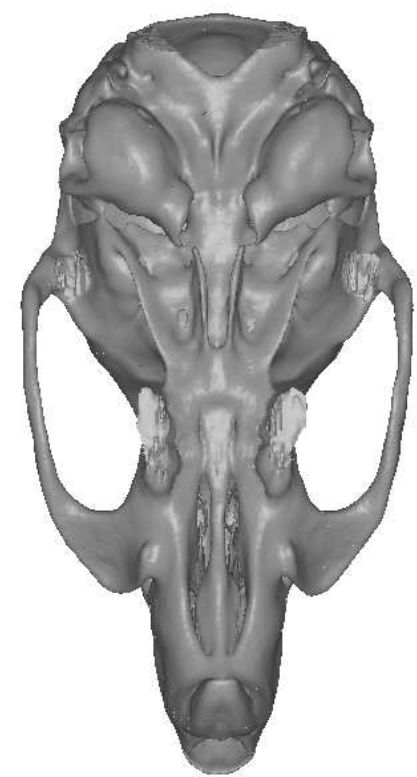

(b)

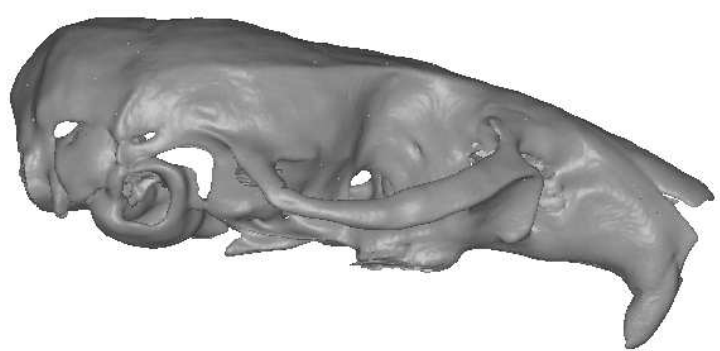

(d)

Figure 7. Second and fifth mode of variation for wild-type mouse SDM. Atlas deformed (a) -2 std. dev. (b) +2 std. dev. along the second principal component. Atlas deformed (c) -2 std. dev. (d) +2 std. dev. along the fifth principal component. Note the different shape of the zygoma in $(a, b)$, the curved form of the calvaria and bending of nose in (c,d). 


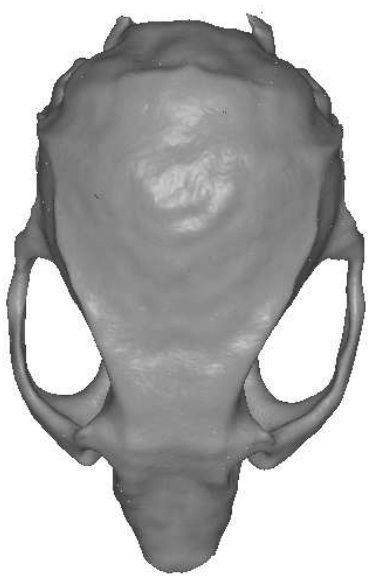

(a)

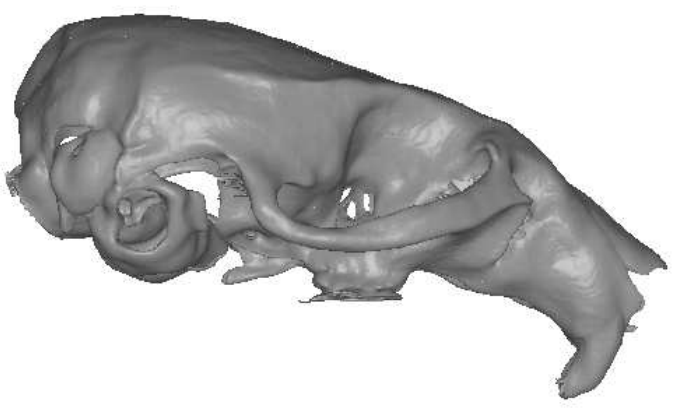

(c)

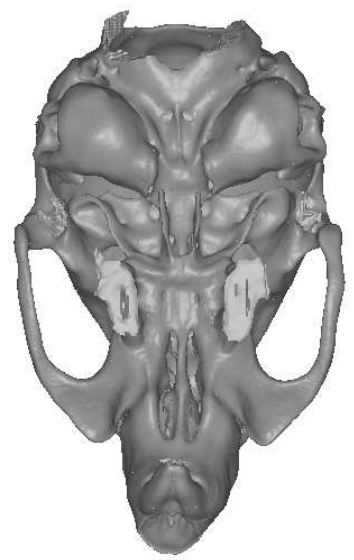

(e)

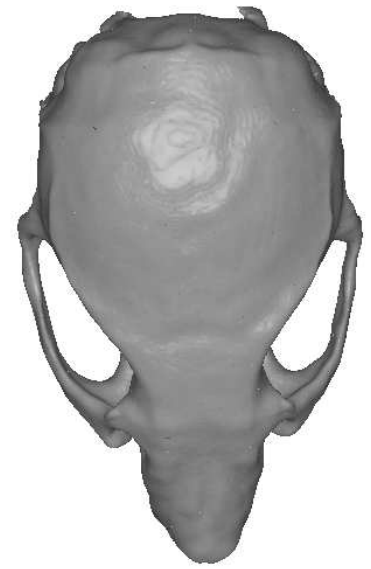

(b)

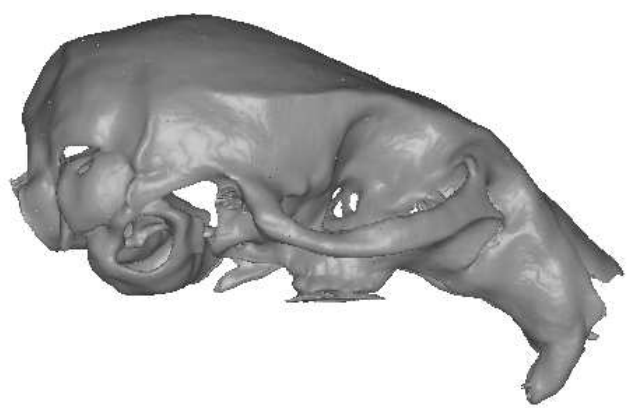

(d)

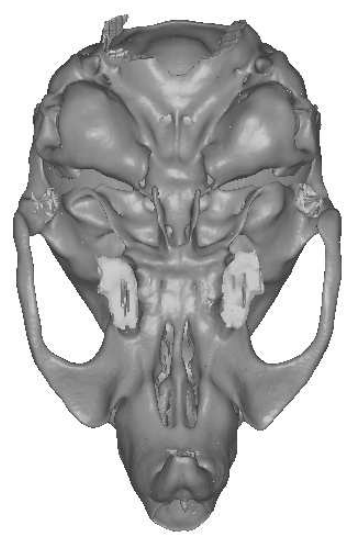

(f)

Figure 8. Second, third and sixth mode of variation for Crouzon mouse SDM. Crouzon atlas deformed (a) -2 std. dev. (b) +2 std. dev. along the second principal component, top view. Crouzon atlas deformed (c) -2 std. dev. (d) +2 std. dev. along the third principal component, side view. Crouzon atlas deformed (e) -2 std. dev. (f) +2 std. dev. along the sixth principal component, bottom view. Note the different shape and symmetry of zygoma in (a,b), different curving of calvaria and nose bending in $(\mathrm{c}, \mathrm{d})$ and nose orientation in $(\mathrm{e}, \mathrm{f})$ 


\section{CONCLUSION}

The combined SDMs confirmed many known traits in the craniofacial anatomy, which already had been reported with respect to human Crouzon subjects. Model A revealed more detailed anatomical differences, while model $\mathrm{B}$ gave the most obvious differences, and in that sense was more intuitive, while some of the local differences were still visible. The within-group models showed similar variation, but the Crouzon model had more extreme variation. Some of the Crouzon modes were asymmetric, which is probably due to asymmetric fusion of sutures, both in terms of the location of sutures and the timing of fusion.

In conclusion, we have built reliable SDMs using the appropriate registration models, which confirm known traits in the Crouzon mouse anatomy. Additionally, traits, which have not, yet, been reported in humans, were discovered and should be further investigated in the future.

\section{ACKNOWLEDGEMENTS}

For all image registrations, the Image Registration Toolkit was used under Licence from Ixico Ltd.

\section{REFERENCES}

1. O. Crouzon, "Dysostose cranio-faciale héréditère," Bull Mem Soc Méd Hôp Paris 33, pp. 545-555, 1912.

2. W. Reardon, R. M. Winter, P. Rutland, L. J. Pulleyn, B. M. Jones, and S. Malcolm, "Mutations in the fibroblast growth factor receptor 2 gene cause Crouzon syndrome," Nat Genet 8, pp. 98-103, 1994.

3. V. P. Eswarakumar, M. C. Horowitz, R. Locklin, G. M. Morriss-Kay, and P. Lonai, "A gain-of-function mutation of fgfr2c demonstrates the roles of this receptor variant in osteogenesis," Proc Natl Acad Sci, U.S.A. 101, pp. 12555-12560, 2004.

4. C. A. Perlyn, V. B. DeLeon, C. Babbs, D. Govier, L. Burell, T. Darvann, S. Kreiborg, and G. Morriss-Kay, "The craniofacial phenotype of the Crouzon mouse: Analysis of a model for syndromic craniosynostosis using 3D MicroCT," Cleft Palate Craniofac. J 43(6), pp. 740-747, 2006.

5. H. Ólafsdóttir, T. A. Darvann, N. V. Hermann, E. Oubel, B. K. Ersbøll, A. F. Frangi, P. Larsen, C. A. Perlyn, G. M. Morriss-Kay, and S. Kreiborg, "Computational mouse atlases and their application to automatic assessment of craniofacial dysmorphology caused by Crouzon syndrome," Journal of Anatomy, 2007 (submitted).

6. D. Rueckert, L. I. Sonoda, C. Hayes, D. L. G. Hill, M. O. Leach, and D. J. Hawkes, "Nonrigid registration using free-form deformations: application to breast MR images," IEEE Trans. on Medical Imaging 18(8), pp. 712-721, 1999.

7. J. A. Schnabel, D. Rueekert, M. Quist, J. M. Blackall, A. D. Castellano-Smith, T. Hartkens, G. P. Penney, W. A. Hall, H. Liu, C. L. Truwit, F. A. Gerritsen, D. L. G. Hill, and D. J. Hawkes, "A generic framework for non-rigid registration based on non-uniform multi-level free-form deformations," Fourth Int. Conf. on Medical Image Computing and Computer-Assisted Intervention (MICCAI '01) 2208, pp. 573-581, 2001.

8. R. H. Davies, C. J. Twining, T. F. Cootes, J. C. Waterton, and C. J. Taylor, "A minimum description length approach to statistical shape modeling," Medical Imaging, IEEE Transactions on 21(5), pp. 525-537, 2002.

9. G. Gerig, M. Styner, D. Jones, D. Weinberger, and J. Lieberman, "Shape analysis of brain ventricles using spharm," IEEE Workshop on Mathematical Methods in Biomedical Image Analysis, 2001. MMBIA 2001. , pp. 171-178, 2001.

10. A. F. Frangi, D. Rueckert, J. A. Schnabel, and W. J. Niessen, "Automatic construction of multiple-object three-dimensional statistical shape models: Application to cardiac modeling," IEEE Trans. on Medical Imaging 21(9), pp. 1151-66, 2002.

11. D. Rueckert, A. F. Frangi, and J. A. Schnabel, "Automatic construction of 3D statistical deformation models of the brain using nonrigid registration," IEEE Trans. on Medical Imaging 22(8), pp. 1014-1025, 2003.

12. S. Kreiborg, Crouzon Syndrome - A Clinical and Roentgencephalometric Study, 1981. Doctorate thesis, Institute of Orthodontics, The Royal Dental College, Copenhagen. 